\title{
Reciclagem de Rejeitos de Poli(tereftalato de etileno) (PET) e de Poliamida (PA) por meio de Extrusão Reativa para a Preparação de Blendas
}

\author{
Caio T. Ferreira, Juliana B. da Fonseca, Clodoaldo Saron \\ Departamento de Engenharia de Materiais, EEL-USP
}

Resumo: O consumo crescente de materiais poliméricos em diversas formas de aplicação leva à produção de uma quantidade enorme de resíduos pós-consumo e pós-industriais com potencial poluidor elevado. A reciclagem mecânica é uma das maneiras mais adequadas para contornar os problemas gerados por estes rejeitos, uma vez que permite conciliar interesse econômico com benefícios ambientais. Neste trabalho foi feito um estudo sobre a reciclagem de rejeitos pós-industriais de poli(tereftalato de etileno) (PET) provenientes da fabricação de mantas de tecido não tecido e de resíduos de poliamida (PA) oriundos de pneus usados na forma de blendas, produzidas por meio de extrusão reativa dos dois resíduos em presença de catalisador. Os resultados obtidos a partir de caracterização térmica e química das blendas evidenciaram a ocorrência de trans-reações entre segmentos de cadeia polimérica dos dois polímeros, possibilitando a compatibilização do sistema. A produção de blendas PET/PA se configura como uma forma adequada para a reciclagem dos rejeitos de PET e de PA.

Palavras-chaves: Reciclagem, PET, poliamida, blendas, extrusão reativa.

\section{Recycling of Wastes from Poly(ethylene tereftalate) (PET) and Polyamide (PA) by Reactive Extrusion for Preparation of Polymeric Blends}

\begin{abstract}
The increasing use of polymeric materials in several applications leads to the production of a high amount of post consume and post industrial wastes with expressive pollutant potential. Mechanical recycling is an important way to decrease the problems caused by these wastes because it allows one to associate economic viability with environmental benefits. In this paper a study was carried out on the recycling of poly(ethylene tereftalate) (PET) wastes from the production of nonwoven fabrics (NWF) and of polyamide (PA) wastes from old tires for production of polymeric blends via reactive extrusion in the presence of trans-reaction catalysts. The results from thermal and chemical characterization indicated trans-reactions between segments of polymeric chains of the two polymers, promoting the system compatibilization. The production of PET/PA blends is an interesting alternative for recycling PET and PA wastes.
\end{abstract}

Keywords: Recycling, PET, polyamide, blends, reactive extrusion.

\section{Introdução}

Os resíduos poliméricos pós-consumo e pós-industriais são fontes de poluição que causam inúmeros problemas ambientais e também representam um desafio para o gerenciamento do lixo em grandes cidades. Com o consumo crescente de polímeros em diversos setores da indústria, estes problemas tendem a ser agravados se não forem adotadas políticas eficientes de reciclagem destes materiais. A produção de polímeros no mundo em 1999 foi de 168 milhões de toneladas, com previsão estimada em 210 milhões de toneladas em $2010^{[1]}$.

Uma parcela considerável dos polímeros termoplásticos é aplicada para a confecção de embalagens que apresentam uma vida útil muito curta e rapidamente se constituem em resíduos sólidos, ocupando boa parte do volume dos aterros sanitários, como é o caso do poli(tereftalato de etileno) $(\mathrm{PET})^{[2,3]}$.

O reaproveitamento de resíduos de PET pode ser feito por meio de reciclagem mecânica (primária ou secundária), química (terciária) e energética (quaternária) ${ }^{[4,5]}$. Entretanto, uma das maiores dificuldades da reciclagem mecânica dos polímeros, principalmente os obtidos por policondensação, é a depreciação de suas propriedades mecânicas devido a processos de degradação por cisão hidrolítica da cadeia polimérica durante a aplicação e, sobretudo, durante o processamento termo-mecânico, quando as condições de umidade não são rigorosamente controladas. $\mathrm{O}$ grau elevado de degradação do polímero leva à produção de rejeitos pós-industriais, como ocorre durante a extrusão de PET reciclado para a produção de mantas de tecido não tecido (TNT).

A característica reativa dos polímeros de condensação pode também ser útil para alguns propósitos, como no caso da compatibilização de blendas. Da mesma forma que ocorre em presença de umidade, as trocas de grupos químicos durante o processamento em altas temperaturas pode ocorrer com grupos do outro polímero de condensação que compõe a blenda polimérica $^{[6,7]}$. As trans-reações, como são chamadas as reações de trocas de segmentos de cadeia em polímeros de condensação, podem ocorrer entre dois poliésteres (transesterificação), duas poliamidas (transaminação) e também entre um poliéster e uma poliamida $^{[6,7]}$.

Os rejeitos de pneus usados representam outra classe de material polimérico com potencial poluidor elevado. O elastômero, principal constituinte dos pneus, encontra-se na forma reticulada, o que dificulta a sua reciclagem por métodos termo-mecânicos. Além do elastômero, os pneus possuem uma quantidade considerável de fibras de poliamida (náilon) como reforço. A poliamida (PA) pode ser separada do pneu por meio de moagem, apresentando-se na forma de lã que ainda contém partículas de borracha agregadas. Os rejeitos de poliamida de pneus usados apresentam um grande potencial de aplicação como material reciclado que ainda não tem sido explorado ${ }^{[8]}$. 
Uma alternativa para a reciclagem mecânica de rejeitos poliméricos que sofreram depreciação de algumas de suas propriedades mecânicas é a produção de blendas. As blendas poliméricas são de grande importância para a indústria, por permitirem a obtenção de produtos com determinadas propriedades sem que haja a necessidade de síntese de novos polímeros e também uma forma de reciclar alguns tipos de polímeros. Outras características são o controle das propriedades a níveis desejáveis e a obtenção em processos relativamente simples e baratos ${ }^{[9-11]}$. A blenda de poliamida-6 (PA-6) com o PET é um dos casos de melhor compatibilidade entre polímeros semicristalinos ${ }^{[10]}$, podendo ser uma alternativa para a reciclagem mecânica de resíduos destes dois polímeros. Retolaza et al. (2005) descrevem a preparação de blendas reativas PA-6/PET em diversas proporções, destacando a sinergia entre os componentes, evidenciada nas propriedades de módulo de elasticidade e resistência à tração das blendas. Esta sinergia é atribuída à formação de copolímeros, mediante a ocorrência de trans-reações entre os componentes que favorecem a interação interfacial na blenda ${ }^{[10]}$.

A proposta deste trabalho foi preparar blendas por meio de extrusão reativa a partir de rejeitos de PET gerados durante a extrusão de "flakes" de garrafas de bebidas no processo de produção de mantas de tecido não tecido (TNT) e de resíduos de poliamida oriundos de pneus, buscando obter evidências da formação de transreações que levam à compatibilização do sistema.

\section{Experimental}

\section{Materiais}

O rejeito de PET na forma de placas com dimensões variadas, o PET reciclado de garrafas de bebidas na forma de "flakes" e o PET virgem grau garrafa na forma de "pellets" foram adquiridos por meio de doação da Fiberweb Bidim Ltda. O rejeito de PET é gerado durante a fabricação por extrusão de mantas de tecido não tecido (TNT) que emprega o PET flake incorporado a uma pequena parcela de PET virgem como matéria-prima. A poliamida (PA) foi obtida por meio de doação da Ecobalto S/A na forma de uma lã que contém partículas de borracha agregadas. Este material é obtido a partir da moagem e separação dos componentes de pneus usados. $\mathrm{O}$ catalisador 2-etilexanoato de estanho (II) foi adquirido da SigmaAldrich com grau de pureza $95 \%$.

\section{Métodos}

Para a caracterização do grau de degradação do rejeito de PET, foram conduzidos ensaios de viscosidade em solução para a determinação da massa molar viscosimétrica do polímero. Para isto, amostras do rejeito de PET, do PET na forma de "flakes" de embalagens recicladas e de PET virgem foram solubilizadas em uma mistura de fenol/1,1,2,2-tetracloroetano na proporção de 60/40 v/v e submetidas aos ensaios de viscosimetria em um viscosímetro Ubbelohde-1B para a determinação do tempo de escoamento das soluções, o qual é empregado para os cálculos de viscosidade intrínseca e da massa molar viscosimétrica do polímero, conforme descrito por Mancini et al. (2004) ${ }^{[12]}$. Os ensaios de viscosimetria foram realizados à temperatura constante de $30^{\circ} \mathrm{C}$ com a imersão do viscosímetro em um banho d' água termoestatizado.

Os rejeitos de PET foram moídos em apenas uma etapa, empregando um moinho de facas piloto da marca MOMESSO até serem reduzidos a partículas com diâmetro inferior a $1 \mathrm{~mm}$, enquanto que os rejeitos de poliamida foram espalhados em uma superfície plana e submetidos a passagem de um campo magnético produzido por um imã, com o objetivo de separar resíduos metálicos presentes no material, oriundos do reforço metálico de muitos pneus, evitando assim, problemas durante a extrusão das blendas.

Na sequência, o PET e a PA foram submetidos à secagem em uma estufa com circulação forçada de ar da marca Quimis modelo 0314N242 durante 4 horas à temperatura de $120^{\circ} \mathrm{C}$. Para a preparação das blendas, a primeira etapa foi a pré-mistura dos dois polímeros nas quantidades de PET/PA de 1500/0 (PET), 1125/375 (PA25), 750/750 (PA50), 375/1125 (PA75) e 0/1500 (PA) em gramas juntamente com 1,5 mL do catalisador 2-etilexanoato de estanho (II) para cada amostra, medidos em uma pipeta volumétrica. $\mathrm{O}$ catalisador tem como função auxiliar na compatibilidade das blendas durante a etapa seguinte de extrusão, por meio da catálise de trans-reações entre o PET e a $\mathrm{PA}^{[13]}$.

Após a pré-mistura, as formulações foram submetidas ao processamento termomecânico em uma extrusora monorosca piloto da marca IMACOM em condições de temperatura de 250, 260, 270 e $270{ }^{\circ} \mathrm{C}$ nas zonas de alimentação, compressão, dosagem e matriz, respectivamente, e velocidade da rosca de $40 \mathrm{rpm}$, com uma rosca especialmente construída para este propósito que contém elementos de mistura do tipo cisalhante (Maddock helicoidal) e distributivo (abacaxi).

Após a peletização, as amostras foram submetidas à secagem em uma estufa com circulação forçada de ar da marca Quimis modelo 0314N242 durante 4 horas à temperatura de $120^{\circ} \mathrm{C}$ e armazenadas para os ensaios de caracterização por TG/DSC simultâneas em um equipamento da marca Setaram modelo LabsysTM, utilizando atmosfera de argônio a uma taxa de aquecimento de $10{ }^{\circ} \mathrm{C} / \mathrm{min}$, partindo da temperatura ambiente até $900{ }^{\circ} \mathrm{C}$. Também foram realizadas análises de FTIR em um equipamento da marca Shimadzu IR-Prestige-21, empregando o método de transmitância com preparação de pastilhas de $\mathrm{KBr}$, nas condições de 32 varreduras e resolução de $4 \mathrm{~cm}^{-1}$. Para as análises de FTIR os materiais foram submetidos a um processo de purificação prévio por meio de dissolução e re-precipitação.

A purificação consistiu inicialmente na dissolução das amostras em fenol/1,1,2,2-tetracloroetano 60/40 v/v para obter uma solução polimérica na concentração aproximada de $5 \% \mathrm{~m} / \mathrm{v}$. Na sequência, a solução contendo partículas em suspensão foi filtrada em papel de filtro. Após, foi acrescentado etanol à solução até que fosse verificada a formação de um precipitado branco, correspondendo ao polímero. Após um período em repouso, o precipitado depositouse no fundo do frasco e a solução resultante foi cuidadosamente retirada com o auxílio de uma pipeta de Pasteur. O precipitado foi novamente lavado com etanol e posto para secar ao ar livre para subsequentes análises de FTIR. O objetivo principal da purificação do PET, da PA e das blendas foi a separação do negro de fumo destes materiais para permitir a obtenção de espectros de FTIR sem a interferência de bandas deste aditivo. Por se tratar de um material insolúvel, o negro de fumo tende a permanecer na forma de particulado na solução, sendo retido no filtro por onde passa a solução antes da adição de etanol.

\section{Resultados}

\section{Massa molar viscosimétrica do PET}

A cisão da cadeia polimérica por hidrólise é um processo de degradação relevante tanto para o PET como para as poliamidas. Entretanto, devido à natureza das ligações, os grupos ésteres do PET são mais susceptíveis à hidrólise do que os grupos amidas das poliamidas $^{[14]}$.

Para se ter uma noção mais clara do estado de degradação em que se encontra o rejeito de PET, na Tabela 1 é feita uma comparação entre a viscosidade intrínseca e massa molar viscosimétrica do 
Tabela 1. Resultados de viscosimetria.

\begin{tabular}{lccc}
\hline & \multicolumn{3}{c}{ Materiais } \\
\cline { 2 - 4 } & $\begin{array}{c}\text { Rejeito de } \\
\text { PET }\end{array}$ & $\begin{array}{c}\text { PET } \\
\text { Flake }\end{array}$ & $\begin{array}{c}\text { PET } \\
\text { virgem }\end{array}$ \\
\hline Viscosidade intrínseca & 0,49 & 0,87 & 1,76 \\
Massa molar (g.mol $\left.{ }^{-1}\right)$ & 11.064 & 26.549 & 80.647 \\
\hline
\end{tabular}

rejeito de PET, do PET na forma de flake, obtido da moagem de garrafas de bebidas e usado como matéria-prima no processo que resulta a formação do rejeito, e do PET virgem, o qual não foi submetido a processamentos termo-mecânicos.

Nota-se que a viscosidade intrínseca do PET flake sofre uma diminuição considerável quando comparada à do PET virgem, resultando no valor de massa molar viscosimétrica em cerca de um terço à do PET virgem. Esta redução de massa molar é devida às etapas de processamento empregadas para a conformação do polímero em garrafas de bebidas e às condições que o material foi submetido durante a sua aplicação e após o seu descarte. Verifica-se que o rejeito de PET também sofre uma redução drástica de massa molar quando comparado ao seu precursor, o PET flake. Neste caso, a degradação do polímero por cisão de cadeia é resultante exclusivamente da etapa de extrusão para a produção das mantas de TNT, indicando que a secagem prévia pode não estar sendo efetuada com o devido rigor necessário.

\section{Análise térmica simultânea TG/DSC}

Na análise térmica simultânea TG/DSC, uma única amostra é analisada e ambos os dados de TG e DSC são obtidos simultaneamente à medida que a amostra é aquecida, o que permite uma associação mais adequada entre os resultados. Na Figura 1 estão apresentados os resultados referentes ao comportamento de perda de massa em função de temperatura obtido por termogravimetria das blendas PET/PA e dos componentes PET e PA.

Percebe-se que as amostras se decompõem praticamente em um único estágio em torno de $400{ }^{\circ} \mathrm{C}$, permanecendo uma quantidade pequena de resíduo ao final da análise. A Figura 2 apresenta uma ampliação da região próxima a $440{ }^{\circ} \mathrm{C}$, na qual é possível notar com mais clareza as diferenças de decomposição entre os materiais.

Nota-se que o PET é o material que apresenta a maior temperatura inicial de decomposição térmica, em torno de $388^{\circ} \mathrm{C}$, enquanto que a temperatura inicial de decomposição térmica mais baixa é observada para a amostra PA, em torno de $300{ }^{\circ} \mathrm{C}$. Para as blendas, a temperatura inicial de decomposição térmica é intermediária ao dos componentes puros, aumentando à medida que o teor de PET na blenda também aumenta, apresentando valores de 319,336 e $371{ }^{\circ} \mathrm{C}$ para as blendas PA75, PA50 e PA25, respectivamente. Deste modo, o PET é o material que apresenta a maior estabilidade térmica, enquanto que as blendas possuem um comportamento intermediário, diminuindo a estabilidade à medida que o teor de PET diminui até atingir o valor mínimo para a PA.

Com relação aos resultados de DSC, a Figura 3 apresenta uma comparação entre as curvas de DSC dos diferentes materiais, cujos valores de fluxo de calor foram normalizados pela massa das amostras e as curvas foram deslocadas ao longo do eixo das ordenadas para facilitar a visualização. Os valores de área e máximo do pico de fusão são apresentados na Tabela 2.

Percebe-se que todas as amostras apresentam um sinal de absorção de calor próximo a $250{ }^{\circ} \mathrm{C}$ que representa a fusão dos materiais. O sinal mais intenso é verificado para o PET, cujo máximo encontra-se a $254{ }^{\circ} \mathrm{C}$. Para a poliamida, o pico de fluxo de calor endotérmico apresenta-se bem menos intenso e com máximo de absorção em $248{ }^{\circ} \mathrm{C}$. No caso das blendas, a área do pico de

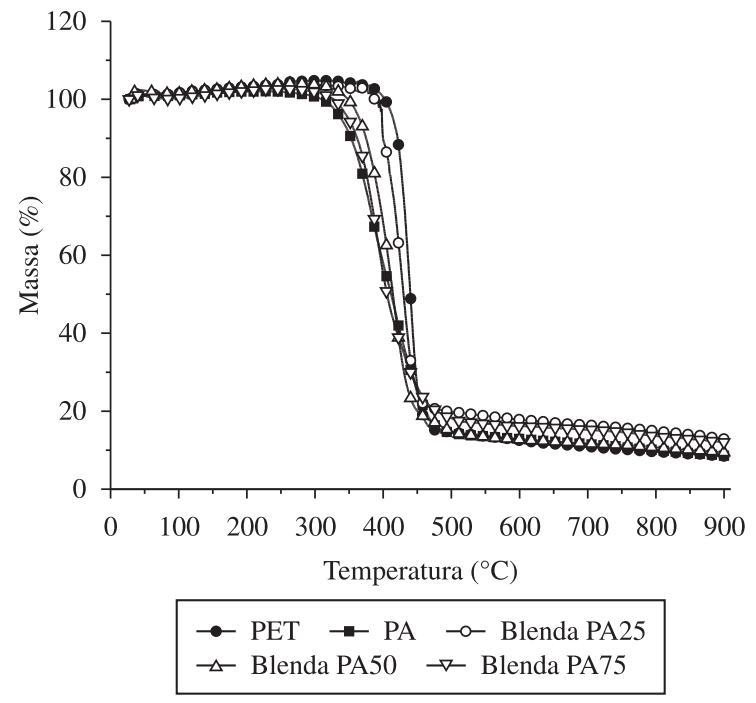

Figura 1. Curvas termogravimétricas: PET (-•-), PA (-ø-), Blenda PA25 $(-\circ-)$, Blenda PA50 (- $\Delta-)$ e Blenda PA 75 (- $\nabla-)$.

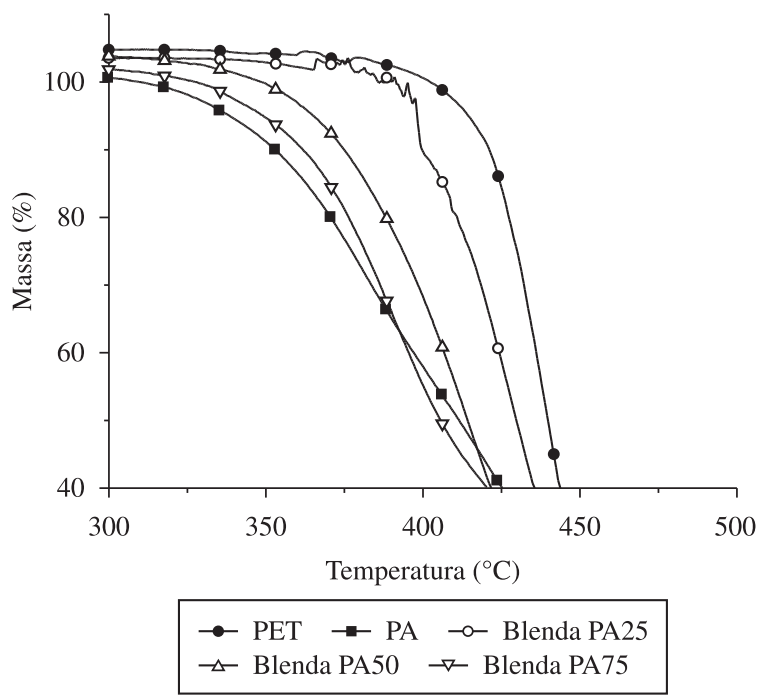

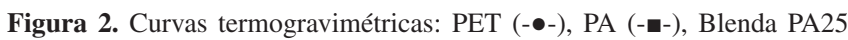
$(-\circ-)$, Blenda PA50 (- $\Delta$-) e Blenda PA $75(-\nabla-)$.

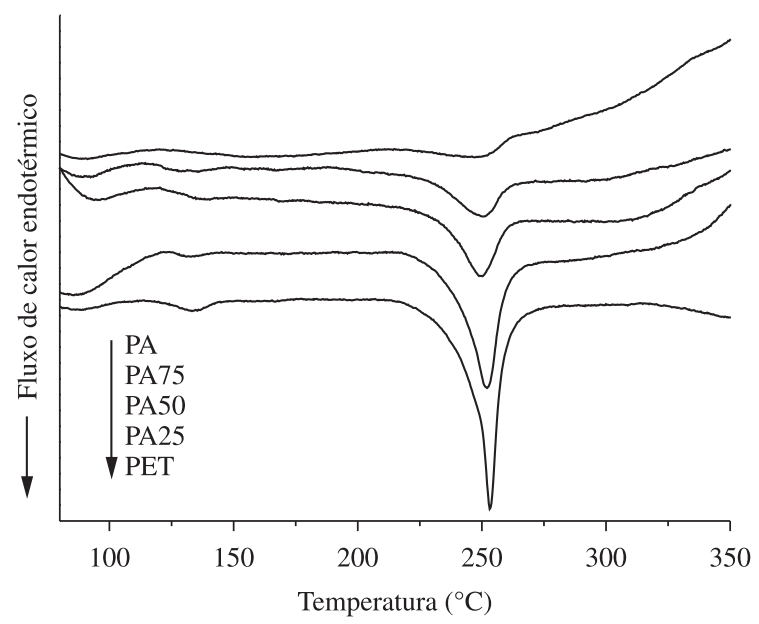

Figura 3. Curvas de DSC para os materiais: PET, PA, Blenda PA25, Blenda PA50 e Blenda PA 75. 
Tabela 2. Temperatura e intensidade da fusão dos materiais.

\begin{tabular}{ccc}
\hline Amostra & $\begin{array}{c}\text { Área do pico de fusão } \\
\text { (U.A.) }\end{array}$ & $\begin{array}{c}\text { Máximo de intensidade do } \\
\text { pico de fusão }\left({ }^{\circ} \mathbf{C}\right)\end{array}$ \\
\hline PA & 1,27 & 248 \\
PA75 & 2,35 & 249 \\
PA50 & 2,86 & 250 \\
PA25 & 5,71 & 252 \\
PET & 6,92 & 254 \\
\hline
\end{tabular}

fusão diminui à medida que aumenta o teor de PA na blenda. Isto significa que no PET a fração de fase cristalina é bem maior do que na PA. Observa-se ainda que nas blendas não existem evidências de dois picos de fusão relativos a cada componente, mas sim de um único pico que sofre deslocamento de temperatura de máximo de intensidade do pico no sentido do componente mais abundante na blenda. Ou seja, se considerarmos o componente PET apenas, a temperatura de fusão da blenda se desloca para valores menores à medida que sua porcentagem na blenda diminui.

Os resultados de DSC evidenciam a ocorrência de interação entre os componentes PET e PA que pode ser do tipo intermolecular ou estar relacionada à formação de copolímeros devido às possíveis trans-reações entre o PET e a PA. Os copolímeros formados apresentam segmentos dos dois polímeros da blenda e cada segmento tem maior afinidade química pela fase rica no polímero correspondente, promovendo maior interação interfacial e consequente compatibilização do sistema. Alguns catalisadores como os do tipo organo-estanho podem aumentar o rendimento de formação de copolímeros, melhorando a compatilidade da blenda $^{[6,7]}$.

\section{Espectroscopia no Infravermelho por Transformada de Fourier (FTIR)}

$\mathrm{Na}$ Figura 4 é feita uma comparação entre os espectros de transmitância dos materiais previamente purificados.

Observa-se que os espectros apresentaram bandas distintas entre si que podem ser comparadas com mais detalhes na Figuras 5 e 6.

A Figura 5 apresenta a comparação entre os espectros na região entre 3700 e $2700 \mathrm{~cm}^{-1}$. Em torno de $2900 \mathrm{~cm}^{-1}$ as bandas observadas em todos os materiais são relativas a estiramentos de ligações $\mathrm{C}-\mathrm{H}^{[15]}$. Na poliamida ocorre uma banda em $2855 \mathrm{~cm}^{-1}$ que diminui relativamente de intensidade nas blendas à medida que o teor de poliamida no material diminui, podendo servir de parâmetro para a quantificação da composição da blenda. Uma banda larga e intensa com máximo de absorção em $3400 \mathrm{~cm}^{-1}$ pode ser verificada no espectro do PET, sendo atribuída à absorção de grupos hidroxilas livres de água contida no polímero ${ }^{[16]}$. A reta que se inicia no ponto de mínimo desta banda também passa pelo mínimo de uma banda existente no espectro da poliamida. Esta banda, juntamente com a banda com máximo de absorção em $3300 \mathrm{~cm}^{-1}$, são características dos grupos N-H das poliamidas. Nas blendas PA25 e PA50 verificase um deslocamento da banda de N-H em $3400 \mathrm{~cm}^{-1}$ para um número de onda mais alto. Este deslocamento não pode ser atribuído simplesmente pela composição das blendas e indica a formação de copolímeros, mediante a ocorrência de trans-reações entre o PET e a PA durante a extrusão reativa do material que resulta na formação de grupos químicos na cadeia polimérica que provocam o deslocamento das bandas.

Na Figura 6 é representada a comparação entre os espectros na região entre 1500 e $2000 \mathrm{~cm}^{-1}$.

A principal banda nesta região refere-se ao grupo carbonila com absorção próxima a $1700 \mathrm{~cm}^{-1}$, que representa a banda de maior intensidade no espectro de FTIR. Tanto o PET como a PA apresentam grupos carbonilas. Entretanto, o ambiente químico em que estas

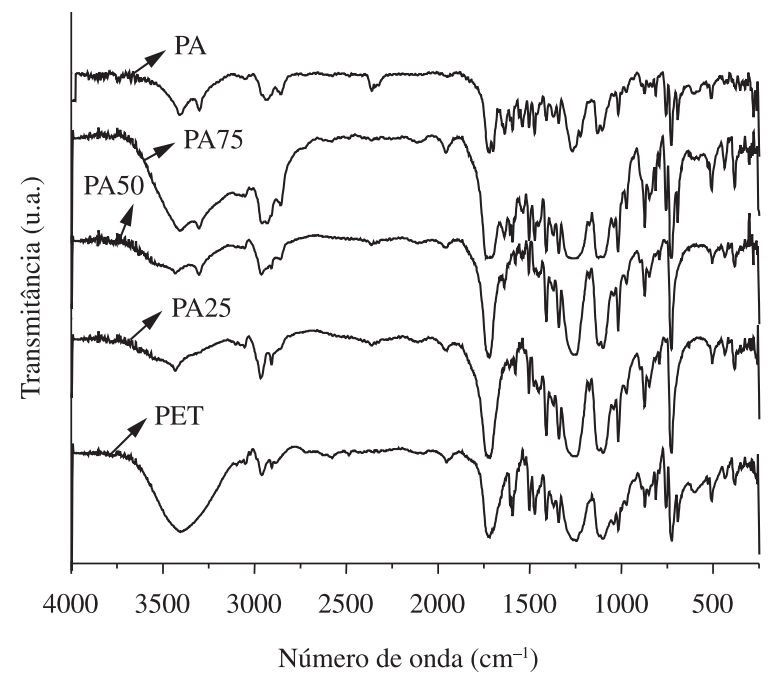

Figura 4. Comparação entre espectros de FTIR dos polímeros.

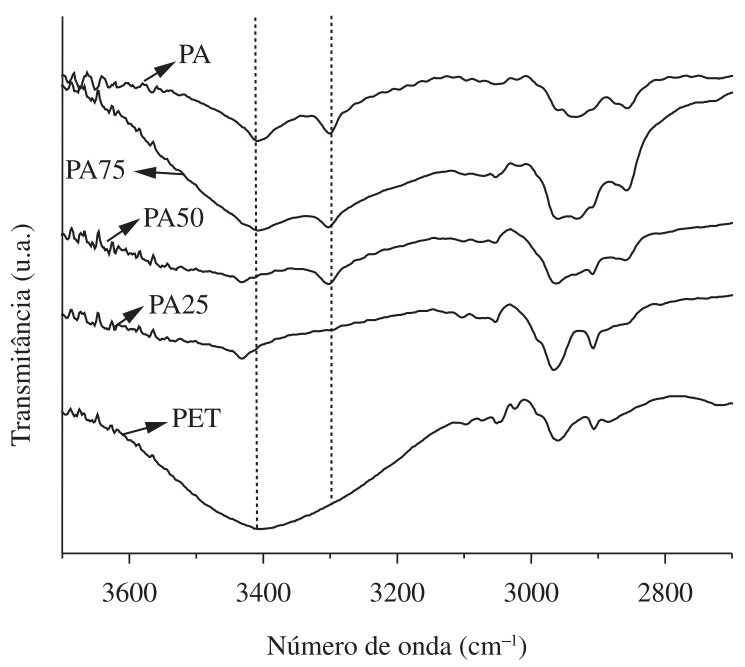

Figura 5. Comparação entre espectros de FTIR dos polímeros (região entre 3700 a $2700 \mathrm{~cm}^{-1}$ ).

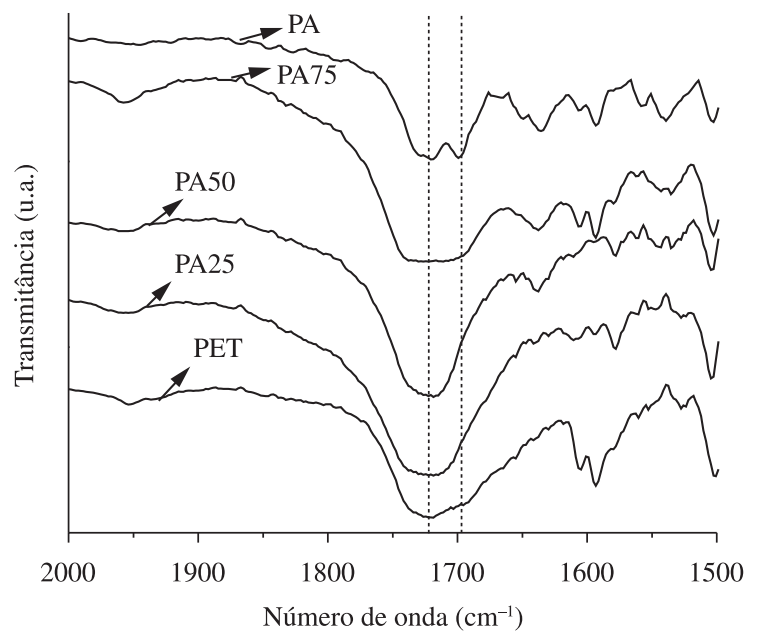

Figura 6. Comparação entre espectros de FTIR dos polímeros (região entre 2000 a $1500 \mathrm{~cm}^{-1}$ ). 


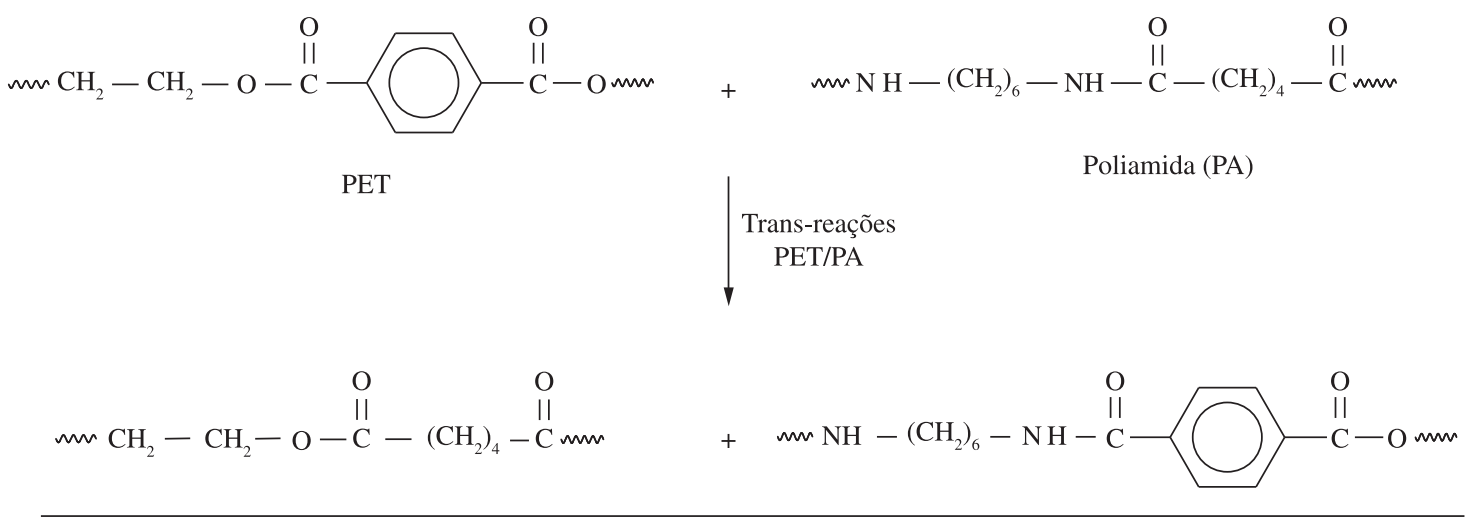

Copolímeros

Figura 7. Formação de copolímeros após trans-reações PET/PA.

bandas se encontram provocam alterações de perfil e deslocamentos nestas bandas, como no caso da poliamida que apresenta a banda duplicada, enquanto no PET uma única banda é observada. Nas blendas, o perfil das bandas de carbonila e das bandas na região entre 1500 e $1700 \mathrm{~cm}^{-1}$ apresentam características que as distinguem do PET e da PA, podendo também indicar a ocorrência de mudanças químicas nos materiais como trans-reações.

A Figura 7 ilustra as prováveis trans-reações que ocorrem entre os polímeros PET e PA que são caracterizadas pelas trocas de segmentos dos dois polímeros para a formação de copolímeros que tendem a compatibilizar a blenda.

\section{Conclusões}

Os rejeitos de PET obtidos do processo de extrusão para a preparação de mantas de TNT apresentam grau elevado de degradação com massa molar bastante reduzida. Estes rejeitos quando combinados por meio de extrusão reativa com rejeitos de poliamida obtidos de pneus usados formam blendas que apresentam copolímeros gerados por trans-reações entre segmentos de cadeia polimérica dos dois componentes. A ocorrência de trans-reações entre o PET e a PA, evidenciada principalmente nas blendas com teores de 25 e $50 \%$ de PET, tende a favorecer a compatibilização das blendas, tornando possível a reciclagem mecânica destes dois rejeitos poliméricos.

\section{Agradecimentos}

- Ao CNPq - processo 471218/2007-3;

- À FAPESP - processo 2007/07676-9;

- À Fiberweb Bidim Ltda; e

- À Ecobalto S/A.

\section{Referências Bibliográficas}

1. Goto, M. - Supercrit. Fluids., 47, p.500 (2009).

2. Romão, W.; Spinacé, M. A. S. \& De Paoli, M. A. - Polímeros, 19, p.121 (2009).

3. Matos, T. F. L. \& Schalch, V. - Polímeros, 17, p.346 (2007).

4. Alonso, J. G.; Molina, E. C.; Muniz, E. C.; Rubira, A. F. \& Carvalho, G. M. - Polímeros, 15, p.27 (2005).

5. Di Souza, L.; Torres, M. C. M. \& Filho, A. C. R. - Polímeros, 18, p.334 (2008).

6. Wang, X. C.; Zheng, Q. \& Yang, G. S. - J. Polym. Sci. B, 45, p.1176 (2007).

7. Huang, D. H.; Woo, E. M. \& Lee, L. T. - Colloid Polym. Sci., 284, p.843 (2006).

8. Anthony, W. S. - Appl. Eng. Agriculture., 22, p.563 (2006).

9. Chiou, K. \& Chang, F. - J. Polym. Sci. B., 38, p.23 (2000).

10. Retolaza, A.; Eguiazábal, J. I. \& Nazábal, J. - J. Appl. Polym. Sci., 97, p.564 (2005).

11. Qu, C.; Yang, H.; Liang, D.; Cao, W. \& Fu, Q. - J. Appl. Polym. Sci., 104, p.2288 (2007).

12. Mancini, S. D.; Matos, I. G. \& Almeida, R. F. - Polímeros, 14, p.69 (2004).

13. Mbarek, S.; Jaziri, M. \& Carrot, C. - Polym. Eng, Sci., 46, p.1378 (2006).

14. Kiliaris, P.; Papaspyrides, C. D. \& Pfaendner, R. - Polym. Degrad. Stab., 94, p.389 (2009).

15. Silverstein, R. M.; Webster, F. X. \& Kiemle, D. - "Spectrometric identification of organic compounds", John-Wiley, New York (2005).

16. Sammon, C.; Yarwood, J. \& Everall, N. - Polymer., 41, p.2521 (2000).

Enviado: $22 / 05 / 10$

Reenviado: 07/08/10

Aceito: $21 / 09 / 10$

DOI: $10.1590 /$ S0104-14282011005000029 